DOCTRINA

\title{
La «neutralidad» del crimen de guerra de violación sexual en el derecho penal internacional
}

\author{
The «neutrality» of the war crime of rape in International Criminal Law
}

\author{
Rita del Pilar Zafra y Pamelhy Valle \\ Pontificia Universidad Católica del Perú
}

\begin{abstract}
RESUMEN En el caso contra Jean Pierre Bemba, la Corte Penal Internacional establece que los elementos del crimen de guerra de violación son neutrales respecto al género. El presente artículo tiene como objetivo probar si es que dicha afirmación es correcta. Para tal efecto, se analizará la definición de género en el estatuto de Roma y se tomará en consideración la forma en la que el derecho internacional y la doctrina lo interpretan. En ese sentido, se busca visibilizar las experiencias de violencia sexual de las personas que se identifican como LGBT en el contexto de los conflictos armados.
\end{abstract}

PALABRAS CLAVE Derecho penal internacional, Corte Penal Internacional, diversidad de género, violencia sexual, crimen de guerra.

ABSTRACT In the case against Jean Pierre Bemba, the International Criminal Court established that the elements of the war crime of rape are gender neutral. The present article seeks to prove whether that assessment is correct. With that objective, the analysis will be based on the regulation of the notion of gender under the Rome Statute, but, especially, under International Law in general, as well as its development within doctrine. The article therefore aims at shedding some light into the experiences of LGBT people in relation to sexual violence in the context of an armed conflict.

KEYWORDS International criminal law, International Criminal Court, gender diversity, sexual violence, war crime. 


\section{Introducción}

El derecho penal internacional tiene sus orígenes en los procesos seguidos contra los criminales de guerra del Eje en los Tribunales Militares Internacionales de Núremberg y del Lejano Oriente. En los setenta años que han transcurrido desde dichos acontecimientos, el derecho penal internacional se ha desarrollado considerablemente.

A nivel institucional, se crearon varios tribunales internacionales para juzgar crímenes internacionales (los Tribunales ad hoc, por ejemplo) y también tribunales mixtos. Entre los desarrollos más importantes y relativamente más recientes se encuentra la creación de la Corte Penal Internacional (CPI), que tiene naturaleza permanente.

A nivel sustantivo, se puede mencionar la evolución del concepto de crimen de lesa humanidad, que elimina el requisito de la conexión con un conflicto armado, y, en lo relativo a los crímenes de guerra, el reconocimiento de que pueden ser perpetrados en el contexto de un conflicto armado no internacional. Asimismo, se reconoció la criminalidad de otras conductas no contempladas en aquellos primeros estatutos. En este punto, se destaca la visibilización de los distintos tipos de violencia sexual a partir de la década de los noventa, gracias al trabajo de los antes mencionados Tribunales ad hoc para la antigua Yugoslavia y para Ruanda (TPIY y TPIR, respectivamente). Se reconoció, entonces, un fenómeno que siempre ha existido en contextos de conflicto armado.

No obstante, el reconocimiento de los distintos tipos de violencia sexual dentro de los conflictos armados, incluida la violación, no se dio de manera uniforme. Gracias a la influencia de la lucha por el reconocimiento de la violencia sexual contra las mujeres, ésta se logró criminalizar y perseguir a nivel internacional. A pesar de esto, la violación sexual contra hombres siguió siendo invisible hasta hace poco y recientemente tribunales como el TPIY y la CPI han establecido condenas por su comisión. La violencia sexual contra personas LGTBI, por el contrario, sigue siendo casi completamente invisible. No ha habido una judicialización de dichos casos y la doctrina tampoco se ha abocado a desarrollar un marco teórico sobre el tema, a pesar de existir evidencia sobre su comisión.

De esta manera, en el presente trabajo buscamos analizar, a través del caso de Jean Pierre Bemba, si la definición de violación sexual existente en el Estatuto de Roma es realmente neutral en lo que respecta al género y, en ese sentido, si dicha definición incluye también a las personas con identidad de género no binaria.

1. Decisión relativa a la apelación de la defensa concerniente a la excepción prejudicial de incompetencia del caso Fiscal contra Dusko Tadic, Tribunal Penal Internacional para la antigua Yugoslavia, 2 de octubre de 1995, párrafo 141. 


\section{Marco conceptual: El «género» como categoría de análisis en los casos de violencia sexual perpetrados en el marco de los conflictos armados}

El empleo del «género» como categoría de análisis implica como primer paso establecer qué se entiende por dicho concepto. Así, se entiende al género como el «conjunto de características asociadas generalmente a la feminidad y masculinidad, que se define de acuerdo a cada sociedad» (No Tengo Miedo, 2016: 13). El género ${ }^{2}$ no es lo mismo que el sexo, pues este último es un «sistema de diferenciación de los cuerpos basado en la presencia de determinados cromosomas, niveles hormonales, órganos reproductivos, órganos genitales externos y características sexuales secundarias» (No Tengo Miedo, 2016: 13).

La definición de género se encuentra íntimamente relacionada con la de «identidad de género» (Comité CEDAW, 2010: párrafo 18), aunque hay que diferenciarlas. Esta última categoría se refiere a la «autopercepción y autoidentificación del género, y puede coincidir o no con lo que se espera socialmente de una persona en función al sexo que se le asignó al nacer» (No Tengo Miedo, 2016:13). La identidad de género se distingue además de la orientación sexual, la cual describe la relación entre el género de la persona y el género de las personas hacia las que siente atracción, ya sea esta afectiva, romántica o erótica (No Tengo Miedo, 2016: 13).

En ese sentido, la identidad de género de una persona puede corresponder a una amplia gama de posibilidades. Puede identificarse como una persona cisgénero, es decir, una persona cuyo sexo asignado al nacer corresponde con su identidad; o una persona trans, esto es, una persona que no se siente identificada con dicho sexo asignado. También puede ser una persona de género no binario, es decir, que no se identifica como hombre ni como mujer o que se identifica con los dos (No Tengo Miedo, 2016: 13-15).

De esta manera, la necesidad de utilizar la noción de «género» como punto de partida del análisis de la violencia que tiene lugar dentro de los conflictos armados se basa en que la misma afecta de manera diferente a grupos distintos de personas. Así, durante los conflictos armados, las diversas formas de violencia ejercidas en tiempos de paz, entre ellas las de naturaleza sexual, se ven agravadas debido a la alteración del orden social establecido (Urban Walker, 2009: 31). Esta última afirmación, no obstante, no deja de reconocer que las afectaciones sufridas por mujeres, hombres y personas LGTB durante un conflicto armado son antecedidas por una larga cadena de situaciones de discriminación que vienen desarrollándose en el contexto de paz,

2. En ese sentido, es entendido como un sistema de organización social que construye la desigualdad en base a la diferencia sexual, con el fin de determinar los roles asignados culturalmente a las personas como propios de un sexo u otro (No Tengo Miedo, 2016: 13). 
generadas por una sociedad que tiene inscrita una visión hetero ${ }^{3}$ cisnormativa ${ }^{4}$ de comprender las relaciones de género.

En efecto, los hechos violentos perpetrados contra mujeres y hombres 5 sólo acentúan los estereotipos socialmente dominantes y las formas de discriminación existentes antes del conflicto (Defensoría del Pueblo de Colombia, 2015: 30), los mismos que legitiman un sistema que concibe las relaciones de género como relaciones de poder entre mujeres y hombres, y entre hombres, que parten de dos instancias: la primera, de la superioridad de lo masculino y subordinación de lo femenino, y la segunda, de la masculinidad hegemónica (o parámetro social para llegar a ser «un verdadero hombre») (Menjívar Ochoa, 2001: 2).

Esta manera de concebir las relaciones de poder forma también parte de la explicación de la violencia sexual dirigida contra las personas LGBT y aquéllas que no se identifican con una de las identidades del binomio hombre/mujer ${ }^{6}$, o, aun haciéndolo, no rechazan el binarismo (Comisión Interamericana de Derechos Humanos, 2015: párrafo 34) y la cisnormatividad. El uso de la violencia contra estas personas responde a «la necesidad de explorar los 'usos de la homofobia' [bifobia y transfobia] como mecanismo de construcción, destrucción y reconstrucción de relaciones sociales de poder» (Serrano-Amaya, 2014: 5), necesidad que se ve también fomentada por un modelo de masculinidad exacerbado. En esa línea, debe reconocerse el carácter constructivo de la violencia (Serrano-Amaya, 2014: 182), esto es, en su papel, por un lado, de eliminador de las transgresiones que atentan el equilibrio de poder y el control sobre los cuerpos, identidades y los roles de género asignados a estos por el patriarcado, y, por otro lado, de refundador del binomio hombre/mujer.

De este modo, las distintas formas de violencia sexual en el contexto de los conflictos armados se basan en el poder y la dominación (Sivakumaran, 2007: 267), por lo que se encuentran relacionadas con la necesidad del mantenimiento y restableci-

3. Hace referencia al sesgo cultural a favor de las relaciones heterosexuales, las cuales son consideradas «normales, naturales e ideales» y son preferidas sobre relaciones del mismo sexo o género (Comisión Interamericana de Derechos Humanos, 2015: párrafo 31). Asimismo, la heteronormatividad parte de un discurso opresor que propone como «válida» $\mathrm{y}$ «natural» una única forma de ser, estar y sentir, altamente adverso a la creación de nuevas categorías (No Tengo Miedo, 2016: 24-25).

4. Hace referencia a la expectativa de que todas las personas a las que se les asignó masculino al nacer siempre crecen para ser hombres y a las que se les asignó femenino, crecen para ser mujeres (Comisión Interamericana de Derechos Humanos, 2015: párrafo 32).

5. Para efectos del presente artículo, cuando hacemos mención a «hombres» $\mathrm{o}$ «mujeres» nos referimos a hombres o mujeres heterosexuales y cisgénero.

6. El cual es concebido como modelo social imperante que considera que el género y el sexo son dos categorías rígidas (masculino/hombre y femenino/mujer), el cual excluye a personas que no se identifican con estas dos categorías (trans no binarios e intersexo) (Comisión Interamericana de Derechos Humanos, 2015: párrafo 34). 
miento de ciertos equilibrios de poder (Sivakumaran, 2007: 267). En ciertas culturas en que las mujeres representan la castidad de la familia y de la propia comunidad (Sivakumaran, 2007: 268-269) y están vinculadas al espacio privado (el hogar) (Montoya, 2011: 21), la violencia sexual perpetrada contra ellas refuerza y transmite la noción de «impotencia masculina», esto es, el fracaso de los hombres de la comunidad en su papel como protectores (Sivakumaran, 2007: 268-269).

Asimismo, esta manera de comunicar dicha impotencia se encuentra presente, incluso es más evidente, en los casos de violencia sexual sufrida por hombres. Como la imagen de «masculinidad» se ha construido en base a la capacidad del hombre de ejercer poder sobre otros, al interior de la familia y de la comunidad, la violencia sexual contra hombres representa no sólo el reforzamiento de la masculinidad del perpetrador, sino también la pérdida de empoderamiento de la víctima (Sivakumaran, 2007: 268-269). En el caso de las personas lesbianas, gays y bisexuales, al ser percibidas como aquéllas que ponen en riesgo el equilibrio de poder del patrón heterosexual y moral imperante, los casos de violencia sexual de los que son víctimas son cometidos con el «fin de 'castigarlos' por traspasar las barreras del género o por cuestionar ideas predominantes con respecto al papel de cada sexo» (Naciones Unidas, 2001: párrafo 17).

El poder que se ejerce sobre mujeres, hombres y personas LGBT durante la ruptura de las hostilidades busca reforzar y reafirmar la posición de dominio preexistente. Así, la extensión de la violencia basada en género en tiempos de paz predispone aquella que se desarrolla en tiempos de guerra (Leatherman, 2012: 34).

En este contexto, una construcción de los elementos del crimen de violación que parte del género como categoría de análisis reconoce que las víctimas no sólo pueden ser mujeres. Ello conllevaría a identificar el término "género» con mujer, lo cual no solo ignora las experiencias de los hombres ${ }^{7}$, sino sobre todo las experiencias de personas que se identifican como LGBT, pues son también de género y, por tanto, deben incluirse dentro de la categoría.

En esa línea, el análisis de los casos de violencia sexual tampoco puede desconocer que los conflictos armados afectan en distinta proporción a mujeres y hombres, y que ambos son víctimas de formas particulares de violencia sexual (Sivakumaran, 2007: 275-276): «Como consecuencia de la inequidad de género, la violencia sexual contra las mujeres no sólo es cuantitativamente diferente (hay mucha más de ella), sino cualitativamente diferente de aquélla que afecta a los hombres» (Gardam, 2014: 5).

En efecto, los hombres también sufren un tipo de violencia particular por su condición de hombres. Por ejemplo, la violación, mutilación genital, obligar a violar otras personas o practicar felación han sido actos asociados a querer atacar el concepto de

7. Véase el artículo de Paul Kirby y Kathleen Kuehnast, «What do we really know about wartime rape?», publicado en Foreign Policy en 2014. Disponible en http://atfp.co/1wzUh73. 
masculinidad de las víctimas (Gorris, 2015) y, en ese sentido, desmoralizar a las sociedades que tienen a la masculinidad como símbolo de su poder (Kapur y Muddell, 2016: 6). Dichos actos provocan que las víctimas se sientan castrados o feminizados (Kapur y Muddell, 2016: 9).

De igual forma, en la violencia sexual contra personas LGBT están también contenidas particulares formas de violencia, pues, por ejemplo:

La experiencia de las mujeres lesbianas no puede igualarse con la de los hombres gays, pues, aunque ambas son estigmatizadas, la primera está atravesada por la experiencia de «ser mujer» en contextos profundamente marcados por la jerarquía de género que privilegia el «ser hombre» (Centro Nacional de Memoria Histórica, 2015: 24).

Cabe destacar en este punto que el enfoque interseccional hace necesario reconocer y analizar la situación cultural, étnica y económica de las víctimas y perpetradores, toda vez que ésta brinda un significado propio al acto de agresión sexual. Así, Grey afirma que para calificar la naturaleza sexual del acto es necesario tomar en cuenta qué es considerado como de naturaleza sexual en la sociedad en la que se produce el crimen (Grey, 2014a: 276), así como la percepción del perpetrador respecto a la pertenencia de la víctima como parte de un grupo social determinado. De esta manera, el análisis de las motivaciones racistas, homofóbicas, xenófobas o sexistas de su comisión no debe realizarse de manera separada, sino conjunta, ya que estos factores interactúan en las estructuras de poder en las que se comete el acto de violencia sexual (Grey, 2014a: 283).

\section{Un análisis del artículo 7.3 del Estatuto de Roma desde la noción de «género»}

En lo que respecta al derecho penal internacional y género, tomaremos como marco el Estatuto de Roma que crea la Corte Penal Internacional ${ }^{8}$. Esto, debido a que, además de ser el primer instrumento convencional multilateral que institucionaliza el derecho penal internacional (Condorelli, 1999: 9, citado en Salmón y García, 2000: 21), es el primer tratado de este derecho que menciona y define la palabra "género». No obstante, la definición que brinda sobre dicho término es muy limitada:

A los efectos del presente Estatuto se entenderá que el término «género» se refiere a los dos sexos, masculino y femenino, en el contexto de la sociedad. El término «género» no tendrá más acepción que la que antecede (artículo 7.3).

8. Adoptado en la ciudad de Roma, Italia, el 17 de julio de 1998, en el marco de la «Conferencia diplomática de plenipotenciarios de las Naciones Unidas sobre el establecimiento de una Corte Penal Internacional». En vigor a nivel internacional desde el 1 de julio de 2002. 
Se pueden apreciar tres puntos esenciales en dicho numeral. En primer lugar, se equivale «género» con «sexo», cuando ambos son categorías diferentes, como se ha explicado con anterioridad en este trabajo. En segundo lugar, se señala que los dos sexos deben ser definidos según el contexto de cada sociedad. Esto último puede tener consecuencias positivas y negativas, como se explicará posteriormente. Y, finalmente, una cláusula de «seguridad», la cual sería redundante, de no ser porque su objetivo es excluir expresamente cualquier otra definición de género que difiera de la establecida en el Estatuto de Roma.

Como afirma Oosterveld, la inclusión de esta definición fue el punto medio que se logró acordar con los países fundamentalistas y religiosos que querían excluir toda mención al género dentro del Estatuto (Oosterveld, 1999: 39-40). La adopción de dicho texto puede ser considerado una victoria en materia de negociación internacional; no obstante, fue mucho lo que se tuvo que sacrificar para poder llegar a un acuerdo. Estas concesiones ocasionaron que el texto tuviera ciertas inconsistencias, lo cual dio origen a sendas críticas.

En principio, hay una obvia confusión entre género y sexo en dicho numeral (Charlesworth, 1999: 394; Rittich y otros, 1999: 207, citado en Oosterveld, 2005: 71). La identificación de género con «femenino y masculino», en lugar de «hombre y mujer», confunde la forma en que la persona se desenvuelve en sociedad de acuerdo a determinados roles y características - género- y la forma de clasificar las características biológicas que presente una persona en «mujer» $\mathrm{u}$ «hombre» - sexo- . Es necesario recalcar, además, que el sexo también es una construcción social, no es un hecho biológico innato (Comisión Interamericana de Derechos Humanos, 2015), más bien es la manera en que la sociedad asigna una categoría, ya sea masculino o femenino, a un cuerpo tomando en consideración sus caracteres genitales.

Aunque algunos autores han afirmado que la referencia al contexto social en el citado numeral es positiva, puesto que reconoce el elemento cultural de la construcción del género -o lo que erróneamente se entiende como género en el Estatuto-, otros afirman que se trata de un esfuerzo para encapsular la concepción de hombre y mujer dando como cierto el concepto existente en cada sociedad (Cossman, 2002: 284, citado en Oosterveld, 2005: 71, 74).

Entre los argumentos en contra, tenemos los de Charlesworth, Moshan, Chinkin y Freedman, quienes critican que únicamente el género sea concebido como un constructo social y no también el sexo, cuando ambos lo son (Oosterveld, 2014). Por otro lado, autores como Chinkin argumentan que dicha definición excluye los crímenes motivados por la orientación sexual de la víctima (Oosterveld, 2014: 569). Ambas posiciones son críticas al carácter limitativo y excluyente de la definición de género según el Estatuto de Roma.

Por otra parte, entre los argumentos a favor de una interpretación inclusiva del referido numeral, se encuentran los de Copelon, quien ha afirmado que en una defini- 
ción amplia de género también puede incluirse la orientación sexual, lo que permite la criminalización de actos de violencia sexual que la tengan como motivación (Oosterveld, 2005: 77-79; 2014: 570-571). No obstante, nosotras no estamos de acuerdo con dicha postura, debido a que, como bien lo afirma la Comisión Interamericana de Derechos Humanos (CIDH), se trata de conceptos distintos, independientes el uno del otro (Comisión Interamericana de Derechos Humanos, 2015: párrafo 19). No se trata de nociones intercambiables. Afirmar esto ocasiona más confusiones en detrimento de la protección que se quiere llegar a ofrecer.

A nuestra interpretación, aún si se considerara que existiese un reconocimiento a la naturaleza de construcción social del género o del sexo en dicho numeral, la restricción a las categorías de "femenino o masculino» impide cualquier intento de realizar una interpretación idónea conforme al concepto de género y sexo aceptados por la doctrina. El entendimiento de género adoptado en el Estatuto de Roma corresponde a una concepción binaria de género, en que las personas sólo podrían identificarse con uno de los dos géneros del binomio hombre/mujer. En consecuencia, se negaría la diversidad de identidades con las que las personas se identifican, más allá de dicho binomio: trans femeninas, trans masculinas, no binarias y andróginas, entre otras.

Así, lo cierto es que, más allá de dicha discusión, bloquea cualquier posibilidad de incluir los crímenes cometidos contra personas que poseen una identidad de género no binaria. Aunque dentro de las categorías de hombre y mujer sí podrían ser incluidas algunas personas que se identifican como trans femeninas o masculinas, no lo harían personas que se identifican fuera del binomio hombre/mujer, por lo tanto, las experiencias específicas de estas personas no serían consideradas.

El 30 de junio de 2014, la Oficina del Fiscal de la CPI publicó su «Policy paper on sexual and gender-based crimes», que es un instrumento - no jurídicamente vinculante- en el que se manifiesta el entendimiento del término género dentro su labor en la CPI. En dicho documento, se define género y sexo de la siguiente manera:

Gender: «Gender, in accordance with article 7.3 of the Rome Statute ('Statute') of the ICC, refers to males and females, within the context of society. This definition acknowledges the social construction of gender, and the accompanying roles, behaviours, activities, and attributes assigned to women and men, and to girls and boys».

Sex: «Sex refers to the biological and physiological characteristics that define men and women» (Oficina del Fiscal de la Corte Penal Internacional, 2014: 3).

Como se observa, la Fiscalía de la CPI adopta una definición que acoge la naturaleza de construcción social del género. No obstante, aún se enmarca en una perspectiva muy estrecha de interpretación, esto es, dentro del esquema binario de género, que sólo reconoce la femineidad y la masculinidad como únicas realidades (No Tengo Miedo, 2016: 26). En ese sentido, aunque haga un esfuerzo de distinguir el concep- 
to de género y de sexo, continúa apostando por entender que el género sólo existe al servicio de la concepción binaria del sexo (Butler, 2002: 182).

Así, y habiendo establecido las limitaciones que el concepto de género presenta en el marco normativo del derecho penal internacional actual, corresponde analizar cuál es el concepto de "género», «identidad de género» $\mathrm{y}$ «sexo» existentes en el marco del derecho internacional de los derechos humanos. Esto, tomando en consideración que, en su artículo 21, el Estatuto de Roma reconoce como parámetro interpretativo, en defecto del propio Estatuto y de las Reglas de Procedimiento y Prueba, las normas de otras ramas del derecho internacional relevantes. Por lo tanto, en dicho artículo se especifica que, por ejemplo, podrán aplicarse los principios y normas del derecho internacional, incluidos los principios establecidos del derecho internacional de los conflictos armados.

Asimismo, se afirma que:

La aplicación e interpretación del derecho de conformidad con el presente artículo deberá ser compatible con los derechos humanos internacionalmente reconocidos, sin distinción alguna basada en motivos como el género, definido en el párrafo 3 del artículo 7, la edad, la raza, [...] u otra condición (artículo 21 del Estatuto de Roma).

No podemos terminar este apartado sin mencionar que, en el citado policy paper, la Fiscalía destaca que el artículo 21 dispone que se debe interpretar el término "género» del artículo 7.3 de conformidad con los derechos humanos internacionalmente reconocidos (Oficina del Fiscal de la Corte Penal Internacional, 2014: párrafos 26-27). No obstante, ambos artículos, aunque amplían el marco del derecho al que se puede recurrir, más allá de las normas propias del Estatuto de Roma, aún restringen la noción de género a aquella definida en el artículo 7.3.

\section{El concepto de «género» en el derecho internacional}

La Declaración de Beijing y la Plataforma de Acción de Beijing de 1995 brindan un acercamiento al término "género» a nivel internacional. Para ello, ambos documentos se remiten al Anexo 4, el cual específica:

En consecuencia, el grupo de contacto reafirmó que la palabra «género» usada en la Plataforma de Acción fue pensada para ser interpretada y entendida de acuerdo con el uso ordinario y generalmente aceptado. El grupo de contacto también acordó que el presente reporte debe ser leído por el presidente de la Conferencia como una declaración del presidente, y que la declaración debe ser parte del reporte final de la Conferencia.

Tomando en cuenta la forma en que la palabra "género» es utilizada a lo largo del anexo, ésta se refiere a los hombres y las mujeres, e incluso podría interpretarse que se refiere sólo a las mujeres, lo que deja de lado además cualquier relación con la 
identidad de género e incluso orientación sexual (no normativas) de las mismas. Así, el concepto «mujer» fue construido desde una visión hetero/cisnormativa, la cual no incluía a la mujer lesbiana, bisexual y trans.

Cabe resaltar, además, que en la negociación del Estatuto de Roma se dejó de lado de manera intencional la definición de género establecida en dichos documentos adoptados en Beijing (Oosterveld, 2005: 63-65). Dicho acuerdo significó que el concepto de género en el derecho penal internacional y en el derecho internacional de los derechos humanos se desarrollase de manera paralela y diferenciada.

A nivel del desarrollo actual del derecho internacional de los derechos humanos, si bien no existe una definición convencional de «género», más allá de la definición contenida en el Estatuto de Roma, sí se puede vislumbrar que existe, o que empieza a existir, una concepción de «identidad de género», que requiere la preexistencia de una concepción de «género» determinada.

Los Principios de Yogyakarta, los cuales son un ejercicio de interpretación de las obligaciones en materia de derechos humanos de los Estados aplicados a la población LGBTI, establecen que la identidad de género se refiere a:

La vivencia interna e individual del género tal como cada persona la siente profundamente, la cual podría corresponder o no con el sexo asignado al momento del nacimiento, incluyendo la vivencia personal del cuerpo [...] y otras expresiones de género, incluyendo la vestimenta, el modo de hablar y los modales (segunda nota al pie de página de los Principios de Yogyakarta, 2007. El énfasis es nuestro).

En dicha definición se resalta la naturaleza de construcción social y cultural del género, así como del sexo (Comisión Interamericana de Derechos Humanos, 2015: párrafos 16-18). La misma ha sido adoptada por la CIDH en su informe sobre violencia contra la población LGTBI en las Américas.

Tanto en los Principios como en el informe ${ }^{9}$ subyace una noción de género que va más allá del binomio masculino/hombre y femenino/mujer.

Asimismo, el reciente nombramiento del experto independiente sobre la protección contra la violencia y la discriminación por motivos de orientación sexual o identidad de género, creado por el Consejo de Derechos Humanos de la Organización de Naciones Unidas y ratificado por la Asamblea General de Naciones Unidas, evidencia que existe cierto consenso sobre la noción de identidad de género a nivel internacional, aunque ésta se encuentre aún en nacimiento. Tampoco se puede obviar que hay una fuerte preocupación internacional por todo intento de socavar el reco-

9. En el informe se sostiene que los sistemas binarios de sexo y género excluyen a aquellas personas que pueden no identificarse dentro de las categorías masculino/hombre y femenino/mujer, como, por ejemplo, algunas personas trans o algunas personas intersexo (Comisión Interamericana de Derechos Humanos, 2015: párrafo 34). 
nocimiento de derechos relacionados con las categorías de «identidad de género» y de «orientación sexual» (preámbulo de Naciones Unidas, 2016).

\section{Avances históricos respecto al crimen de guerra de violación sexual: un primer acercamiento al reconocimiento de la violación contra hombres y la invisibilización de la violación contra personas LGTB}

Lamentablemente, la violencia sexual siempre ha estado presente en la mayoría de los conflictos armados. Respecto a los crímenes juzgados ante los Tribunales Militares Internacionales de Núremberg y Tokio, existe un consenso en la crítica a la falta de inclusión en los cargos y de juzgamiento de los crímenes de violencia sexual en general, y de violación, en específico, que fueron constantes durante los enfrentamientos entre los Aliados y el Eje. Esto se predica, sobre todo, frente a crímenes devastadores como la masacre de Nanking, perpetrada contra la población china de esa ciudad por parte de las fuerzas armadas japonesas, y por las mujeres «confort» proporcionadas también a los soldados japoneses de la población de mujeres coreanas y chinas.

Cabe resaltar que la violencia sexual contra hombres y niños también estuvo presente en la masacre de Nanking (Falk y Wright, 1971: 124; Chang, 1997: 88-89, 95, citado en Sivakumaran, 2007: 264). Sin embargo, en estos contextos, la discusión sobre los posibles casos de violación y otros tipos de violencia sexual contra la población LGTB ni siquiera ha sido planteada de una manera seria y profunda.

El patrón de invisibilización de la violencia sexual contra determinado grupo de personas ${ }^{10}$ durante los conflictos armados también se repitió en el ámbito nacional. Por ejemplo, en el caso del conflicto armado no internacional vivido en Perú de 1980 al 2000, además de cruentos casos de violencia sexual hacia mujeres, también hubo casos de violencia sexual contra hombres. En concreto, se puede señalar el caso de los hechos que tuvieron lugar en el cuartel Los Cabitos, en el cual a los detenidos se les obligaba a desnudarse y electricidad era aplicada a sus genitales (Comisión de la Verdad y Reconciliación, 2003: 82).

Sin embargo, estos hechos no fueron clasificados como actos de violencia sexual, sino como tortura o tratos crueles (Sivakumaran, 2007: 256-257), aunque la desnudez y la manipulación de los genitales tienen una clara connotación sexual.

Asimismo, en el informe final se afirma que la conducta del Movimiento Revolucionario Túpac Amaru (MRTA) estimuló prejuicios contra homosexuales. Así, entre su accionar estuvo el asesinato de un homosexual, cuyo cuerpo fue hallado junto a un cartel que decía "Así mueren los maricones». Este grupo armado justificó la comisión de dichos crímenes bajo el argumento de que «estas lacras sociales» corrompen a los jóvenes (Comisión de la Verdad y Reconciliación, 2003: 432-433).

10. Léase hombres heterosexuales y personas que se identifican como LGBT. 
En el marco del conflicto armado no internacional colombiano, en cambio, la discusión sobre posibles casos de violación contra la población LGTB se ha venido planteando de una manera seria. Se ha reportado una serie de actos violación sexual, calificados como tal, contra lesbianas, gays, hombres y mujeres trans que respondería a una práctica de "castigo» $\mathrm{y}$ "corrección» por su orientación sexual e identidad de género (Centro Nacional de Memoria Histórica, 2015: 246-248). Se habla, en ese sentido, de actos de carácter estratégico ejercidos por los grupos armados o las fuerzas estatales «para instaurar un orden moral que refuerza los órdenes de género y sexualidad hegemónicos» (Centro Nacional de Memoria Histórica, 2015: 251). Al respecto, el Centro Nacional de Memoria Histórica narra, entre otros testimonios, los de una mujer lesbiana (víctima de un grupo de paramilitares), un hombre trans (víctima de un miembro de la guerrilla de las FARC) y de un hombre gay (víctima de un grupo de paramilitares), en ese orden:

Una amiga le dijo que no siguiera molestando porque yo era lesbiana [...]. Eso fue la ofensa más grande para él... [...] me dijo que me iba a enseñar a ser mujer y empezó a golpearme y me violó (Centro Nacional de Memoria Histórica, 2015: 252).

Por mi condición de chico trans, he recibido insultos de parte de paramilitares, [...] fui víctima de violencia sexual [...], durante el momento de la violación siempre me estaban diciendo que yo no era un hombre, que a mí me podían hacer lo que le hacían a cualquier mujer, que el hombre tenía pene y que dónde estaba mi pene [...] (Centro Nacional de Memoria Histórica, 2015: 258).

Me echaron escopolamina y el cuerpo se me durmió [...] ellos me agarraron y me subieron en una camioneta [...]. Luego de eso yo alcanzaba a ver que me tiraron como en un espacio oscuro inmenso [...] ellos me hicieron todas las barrabasadas más grandes del mundo [...]. Me violaron, me pegaron, me ultrajaron (Centro Nacional de Memoria Histórica, 2015: 265).

Punto aparte merece mención el desarrollo jurisprudencial del TPIY y el TPIR ${ }^{11}$, los cuales si bien marcaron un hito en el reconocimiento y castigo de los distintos tipos de violencia sexual cometidas contra mujeres en contextos de conflicto armado, lo cierto es que los distintos actos de naturaleza sexual cometidos contra hombres no fueron considerados como tales, aunque, nuevamente, dichos actos fueran descritos en los casos (Sivakumaran, 2007: 259-261). Así, por ejemplo, el 80\% de 5.00o hombres que se encontraban en Sarajevo fueron violados (Mudrovcic, 2001: 60-76, citado en Sivakumaran, 2007: 263). No obstante ello, encontramos un primer acercamiento al reconocimiento del crimen internacional de violación sexual como una experiencia también de hombres, aunque en esta etapa se estuvo aún muy lejos de reconocer las experiencias de las personas que se autoidentifican como LGBT.

11. En sus estatutos no se consideró a la violación como crimen de guerra ni tampoco como genocidio, pero sí como crimen de lesa humanidad. 
El caso Dusko Tadic es el primer caso analizado por el TPIY y el primero que describe diversos actos de violencia sexual contra hombres, incluida la violación. En la sentencia, si bien se detallan actos de mutilación sexual, sexo oral, felación y desnudamiento forzado contra hombres ${ }^{12}$, no se clasifican tales actos como de índole sexual. Al momento de la calificación, el TPIY consideró que dichos actos eran actos de trato inhumano y que, al ser cometidos en el marco de un conflicto armado como parte de un ataque generalizado o sistemático contra una población civil, constituían crímenes de lesa humanidad ${ }^{13}$.

De igual modo, en el caso conocido como Celebic, Zejnil Delalic, Zdravko Mucic, Hazim Delic y Esad Landzo, ellos fueron acusados de cometer, además de violación sexual ${ }^{14}$ contra mujeres - considerada como tortura siempre que cumplía con los requisitos establecidos en la Convención contra la Tortura y Otros Tratos o Penas Crueles, Inhumanos o Degradantes de $1984-{ }^{15}$, otros actos de clara connotación sexual contra hombres, aunque, otra vez, fueran clasificados como actos de trato inhumano y trato cruel (recogidos en los artículos 2.b y 3 del Estatuto, referidos a «infracciones graves a la Convención de Ginebra de 1949" y «violaciones de las leyes o prácticas de guerra», respectivamente). Dentro de estos actos se encontraba la violación, al haber obligado a dos hombres a cometer felación entre ellos ${ }^{16}$.

A diferencia de los casos contra Tadic y Celebic, en el caso contra Ranko Cesic existe un reconocimiento explícito de que ciertos actos de naturaleza sexual ejercidos contra hombres, como el sexo oral forzado, constituyen violación. En esa línea, el TPIY condenó por violación a Ranko Cesic al haber obligado a dos hermanos musulmanes a cometer felación entre ellos en presencia de otras personas, con lo cual establecieron que este acto de agresión sexual constituía un crimen de lesa humanidad (violación) y un crimen de guerra (trato humillante e inhumano) ${ }^{17}$.

En este punto, cabe preguntarnos si el hecho de que no se haya clasificado, en casi la totalidad de los casos en los que se recogió, actos de violación cometidos contra hombres como crimen internacional, con lo cual fueron tratados como un tipo de violencia sexual invisible, se debe a que la definición de violación adoptada por

12. Sentencia del caso Fiscal con Dusko Tadic y otros, Tribunal Penal Internacional para la antigua Yugoslavia, 7 de mayo de 1997, párrafos 194, 198, 206.

13. Sentencia del caso Fiscal con Dusko Tadic y otros, párrafo 730.

14. Recoge la definición de violación sexual adoptada en el caso contra Jean Paul Akayesu del Tribunal Penal para Ruanda.

15. Sentencia del caso Fiscal con Zejnil Delalic, Zdravko Mucic alias «Pavo», Hazim Delic, Esad Landzo y otros, Tribunal Penal Internacional para la antigua Yugoslavia, 16 de noviembre de 1998, párrafo 493

16. Sentencia del caso Fiscal con Zejnil Delalic, Zdravko Mucic alias «Pavo», Hazim Delic, Esad Landzo y otros, párrafos $26,1.065$.

17. Sentencia del caso Fiscal con Ranko Cesic y otros, Tribunal Penal Internacional para la antigua Yugoslavia, 11 marzo de 2004, párrafos 13, 107. 
los Tribunales ad hoc no permitía considerar como víctimas a hombres y mujeres indistintamente.

El TPIY, en el caso Anto Furundzija, en el que nuevamente se considera la violencia sexual contra mujeres como tortura y se precisa además los elementos de ésta en el contexto de los conflictos armados ${ }^{18}$, establece un concepto de violación estricta. Así, define a la violación como un acto que implica la penetración de la vagina o el ano de la víctima por parte del perpetrador o cualquier otro objeto usado por éste; o de la boca de la víctima por el pene del perpetrador, en que la penetración debe realizarse bajo coacción o amenaza de emplear la fuerza contra la víctima o tercera persona ${ }^{19}$.

En el caso Dragoljub Kunarac, este mismo tribunal condenó a los acusados (Kunarac, Kovac y Vukovic) también por violación sexual contra mujeres, al afirmar que dicho crimen constituía un crimen de lesa humanidad y un crimen de guerra ${ }^{20}$. Asimismo, destacó que los distintos actos de violencia fueron ejercidos con fines discriminatorios: entre los motivos se encontraba la discriminación por género, y estaban dirigidos especialmente a mujeres y niñas vulnerables e indefensas ${ }^{21}$. En este caso, se señaló que el actus reus del crimen de violación es la penetración sexual, aunque sea leve, de la vagina o el ano de la víctima por el pene del perpetrador o cualquier otro objeto empleado por el perpetrador; o de la boca de la víctima por el pene del perpetrador $^{22}$, en que la penetración sexual ocurre sin el consentimiento de la víctima, teniendo en cuenta el contexto en el que se desarrolla ${ }^{23}$. En este último aspecto se diferencia de la definición de violación adoptada en el caso contra Furundzija, pues en esta la penetración sexual requería el empleo de la coacción, fuerza o amenaza contra la víctima o una tercera persona ${ }^{24}$.

El TPIR, en el caso Jean Paul Akayesu, es el primero en ofrecer una definición de violación bastante amplia, pues la concibe como una invasión física de naturaleza sexual cometida contra una persona bajo circunstancias coactivas ${ }^{25}$. En sí, considera como violencia sexual la violación y todo acto de índole sexual ejercido contra una persona bajo circunstancias coactivas. Resalta, además, que la violencia sexual no se

18. Sentencia del caso Fiscal con Anto Furundzija y otros, Tribunal Penal Internacional para la antigua Yugoslavia, 10 de diciembre de 1998, párrafo 162.

19. Sentencia del caso Fiscal con Anto Furundzija y otros, párrafo 185.

20. Sentencia del caso Fiscal con Dragoljub Kunarac, Radomir Kovac, Zoran Vukovic y otros, Tribunal Penal Internacional para la antigua Yugoslavia, 22 de febrero de 2001, párrafos 4, 9-10.

21. Sentencia del caso Fiscal con Dragoljub Kunarac, Radomir Kovac, Zoran Vukovic y otros, párrafo 867. 22. Sentencia del caso Fiscal con Dragoljub Kunarac, Radomir Kovac, Zoran Vukovic y otros, párrafo 437. 23. Sentencia del caso Fiscal con Dragoljub Kunarac, Radomir Kovac, Zoran Vukovic y otros, párrafo 460. 24. Sentencia del caso Fiscal con Dragoljub Kunarac, Radomir Kovac, Zoran Vukovic y otros, párrafo 437. 25. Sentencia del caso Fiscal con Jean-Paul Akayesu y otros, Tribunal Penal Internacional para la antigua Unión Soviética, 2 de setiembre de 1998, párrafo 688. 
limita a la invasión física, dado que puede incluir actos que no impliquen la penetración ni el contacto físico. Se aprecia que, a diferencia de otros casos, aquí se menciona el término «invasión» y no "penetración» para definir la violación sexual; asimismo, que no todo acto de índole sexual requiere un contacto físico. Esto último permitió considerar a la desnudez forzada como violencia sexual. Por último, este Tribunal sostuvo que las mencionadas circunstancias coactivas no necesariamente implican el empleo de la fuerza física, sino que las amenazas, la intimidación, la extorsión, el miedo y ciertas circunstancias como la existencia de un conflicto armado constituyen también formas de coacción ${ }^{26}$.

Nótese, entonces, que la evolución del concepto de crimen de violación, así como el análisis de los casos en la citada jurisprudencia internacional, ha estado principalmente ligada a la experiencia de las mujeres y niñas, y no de los hombres, a pesar de que a partir de la definición de violación adoptada por los Tribunales ad hoc se infiera que, indistintamente, hombres y mujeres podían ser juzgados como perpetradores o protegidos como víctimas. Esto se debió a que lamentablemente era común el empleo del «género» como sinónimo de mujeres para comprender la comisión de todo acto de violencia sexual, a lo que se suma el reforzamiento de estereotipos en narrativas que concebían a la mujer siempre como víctimas y a los hombres como victimarios ${ }^{27}$.

\section{Definición del crimen de guerra de violación en el Estatuto de Roma}

La violación es una de las formas de violencia sexual criminalizadas bajo el Estatuto de Roma, con lo que constituye un crimen de guerra que se puede perpetrar tanto en un conflicto armado internacional como no internacional. Cabe observar que la protección otorgada en los dos contextos es la misma, por lo que se trata de una protección uniforme en todo contexto de conflicto armado.

La definición de dicho crimen incorpora los aportes de la jurisprudencia de los TPIY y TPIR, sobre todo en lo que corresponde a los elementos del actus reus sobre invasión del cuerpo y contextos coercitivos, tal como fue establecido en el antes mencionado caso Akayesu. De esa forma, el Estatuto de Roma es el resultado de todos los avances originados desde los Tribunales Militares Internacionales y, por lo tanto, refleja sus virtudes, pero también sus deficiencias.

En lo que respecta a la práctica, la persecución de la violencia sexual ha sido un tema relegado en los primeros casos que abordó la CPI. En su primer caso contra Thomas Lubanga Dyilo se criticó duramente que la Fiscalía no incluyera crímenes de violencia sexual dentro de los cargos (Tan, 2014; Grey, 2014b: 606). Aunque posteriormente hubo un intento de incluir dichos hechos dentro de algunos de los cargos

26. Sentencia del caso Fiscal con Jean-Paul Akayesu y otros, párrafo 688.

27. Véase el artículo de Paul Kirby y Kathleen Kuehnast en Foreign Policy, antes mencionado. 
presentados (Tan, 2014: 122-123; Grey, 2014b: 606), la Corte rechazó dicha argumentación ${ }^{28}$ (Tan, 2014: 118-119). Es recién en el caso contra Bosco Ntaganda en el cual se presentan y formalizan cargos por crímenes de guerra por violencia sexual ${ }^{29}$. Y, finalmente, es en el caso contra Jean Pierre Bemba cuando se establece una condena por el crimen de guerra de violación sexual.

\section{El caso contra Jean Pierre Bemba: Una oportunidad perdida para el reconocimiento de un concepto de violación inclusivo}

Jean Pierre Bemba fue un comandante del Mouvement de la Liberation du Congo (MLC), grupo armado que protagonizó un conflicto en la República Centroafricana del 2002 al 2003 contra las Fuerzas Armadas Centroafricanas (FACA) (Corte Penal Internacional, 2016).

El 21 de marzo de 2016, Jean Pierre Bemba fue hallado culpable de cinco cargos: asesinato y violación (ambos como crimen de guerra y crimen de lesa humanidad) y del crimen de guerra de pillaje. Por estos crímenes, fue condenado a dieciocho años de privación de libertad, de los cuales se deducirán el tiempo que ha estado en custodia de la CPI durante su juzgamiento. En la actualidad, el caso se encuentra en la etapa de apelación, recurso presentado tanto por la Fiscalía como por la Defensa (Corte Penal Internacional, 2016).

Los jueces encontraron que Bemba era responsable de, entre otros, el crimen de guerra de violación contra mujeres y hombres. Respecto a los actos de violación contra hombres, se encontró que los perpetradores invadieron por la fuerza los cuerpos de $\mathrm{P}_{23^{30}}$ y $\mathrm{P} 69^{31}$ «al penetrar sus [...] anos, y/u otras aperturas del cuerpo con sus penes» $»^{32}$.

$\mathrm{Al}$ realizar el análisis de los elementos objetivos y subjetivos del crimen de violación para la calificación de tales actos, la CPI encontró que:

La Cámara enfatiza que, según los elementos de los crímenes, «el concepto de «invasión» pretende ser lo suficientemente amplio como para ser neutral al género». En consecuencia, «invasión», en el marco legal de la Corte, incluye penetración entre personas del mismo sexo, y abarca víctimas y perpetradores tanto masculinos como femeninos ${ }^{33}$.

28. Sentencia del caso Fiscal Thomas Lubanga Dyilo, Corte Penal Internacional, 14 de marzo de 2012, párrafo 630 .

29. Decisión de confirmación de cargos del caso Fiscal con Bosco Ntaganda, Corte Penal Internacional, 9 de junio de 2014 .

30. Sentencia del caso Fiscal con Jean Pierre Bemba, Corte Penal Internacional, 21 de marzo de 2016, párrafo 494.

31. Sentencia del caso Fiscal con Jean Pierre Bemba, párrafos 498, 501.

32. Sentencia del caso Fiscal con Jean Pierre Bemba, párrafo 633.

33. Sentencia del caso Fiscal con Jean Pierre Bemba, párrafo 100. 
Según la Corte, el concepto de «invasión» es neutro en lo que respecta al género, lo que permite juzgar tanto perpetradores o proteger a víctimas que sean hombres o mujeres. Esta definición es acorde con el antes expuesto desarrollo en los Tribunales ad hoc. No obstante, ¿es eso lo que en realidad significa ser gender-neutral?

La Universidad Berkeley define a dicho término como:

Lenguaje inclusivo para describir relaciones («cónyuge» [spouse] y «pareja» [partner] en vez de "esposo/novio» y «esposa/novia»), espacios (baños inclusivos o de género neural, usados por todos los géneros), pronombres ([they and $\left.z e^{34}\right]$ son pronombres inclusivos y de género neutral) entre otras cosas (Berkley’s Gender Equity Resources Center, 2013).

Nótese que la definición se refiere a todos los géneros, no a los dos géneros. La definición de género que utiliza dicha casa de estudios remite a su vez a la definición de «identidad de género», en la que se resalta que «algunas identidades de género son «mujer», «hombre trans» [transman] y «agénero» [agender], pero hay muchas otras» (Berkeley's Gender Equity Resources Center, 2013). Es decir, dicha inclusividad permite el reconocimiento de identidades de género diferentes a las asociadas al binomio masculino/femenino.

Aunque el afirmar explícitamente (a nivel jurisprudencial) que la penetración entre personas del mismo sexo (de conformidad con el entendimiento de sexo genital según el Estatuto) también es considerada como invasión representa un avance positivo sobre la materia, no es suficiente para incluir a las personas que no se encuentran dentro del concepto binario de género y de sexo en los roles de perpetrador y víctima. Así, la CPI replica el problema que arrastra la redacción del Estatuto mismo y pierde la oportunidad de extender el entendimiento jurisprudencial de víctimas de violación sexual.

Por otro lado, si bien podría afirmarse que los hechos en el caso Bemba, al no recoger testimonios de víctimas LGTB, no obligaban a la CPI a pronunciarse, consideramos que ya habiéndose arriesgado a declarar el concepto de crimen de violación como gender-neutral, hubiera sido una oportunidad clave para visibilizar la posibilidad de que las personas LGTB puedan ser vistas como víctimas y perpetradores de dicho crimen. No obstante lo anterior, lo cierto es que la CPI aún estaría regida por la definición limitativa del artículo 7.3, por lo que las experiencias de aquellas personas con identidades no binarias no podrían ser consideradas.

En ese sentido, hay que recordar que: «Mientras este modelo parece ser neutral respecto al género, se basa de todos modos en nociones de cuerpos y acciones generizados. Entendimientos generizados particulares de cuerpos y sexualidad definen el contenido del daño de la violencia sexual» (Campbell, 2007: 416-417).

34. Formas para «ellos» $\mathrm{y}$ «ellas» intraducibles al castellano. 
Afirmar que la definición de violación es neutral porque sólo lee los cuerpos y no identidades de género de las personas es negar que los cuerpos se encuentran generizados. El Estatuto de Roma limita esta lectura al binomio sexo masculino/sexo femenino. Al excluir todo tipo de identidad de género distinta a la que calce en dicho binomio, se invisibiliza las experiencias de las personas cuyos cuerpos están siendo leídos.

La CPI, en este caso, recoge la percepción de $\mathrm{P}_{23}$ respecto a la experiencia que sufrió como víctima de violación. Al respecto, $\mathrm{P}_{23}$ afirmó que debido a ese hecho la gente de su comunidad lo despreciaba y que él se consideraba un «hombre muerto» ${ }^{35}$. Este testimonio es un aporte importante a efectos de una mayor consciencia sobre la existencia de hombres víctimas de violación por su condición de hombres; para, así, evitar el reforzamiento de narrativas sobre mujeres como víctimas y hombres como perpetradores. De esta manera, la CPI hizo uso del caso para conceptualizar, identificar, describir, calificar y hacer visible la violación sexual contra hombres (Kapur y Muddell, 2016: 27-28).

Hubiese constituido también una oportunidad para abrir la posibilidad de visibilizar la violación hacia personas LGTB como crimen de guerra, lo cual es esencial para lograr a futuro la condena de tal acto y asegurar la inclusión de todas las víctimas, en particular las más invisibles y también vulnerables (Kapur y Muddell, 2016: 27-28).

Se evitaría, así, que únicamente las experiencias de las personas heterosexuales y cisgénero se conviertan en la base de los criterios mediante los cuales la CPI analice y califique los casos de violación u otros actos de violencia sexual como crimen internacional.

\section{Conclusiones}

La definición de «género» en el Estatuto de Roma es bastante limitada. En concreto, se confunde con la de sexo. En segundo lugar, adopta una postura acorde con las concepciones binarias de género y sexo, que excluye a toda la diversidad existente sobre identidad de género.

En el caso Bemba se afirma que la definición de «invasión» es neutral respecto al género. No obstante, el concepto de género está estrechamente vinculado al de identidad de género, por lo que la neutralidad incluye a todos los géneros, no sólo al masculino y al femenino, sino también trans no binarios, andróginos, etcétera.

La CPI perdió la oportunidad de afirmar que las personas LGTB también pueden ser víctimas del crimen de guerra de violación sexual, en razón de la definición neutral antes mencionada, y no sólo abrir esa posibilidad para los hombres. De esa manera, se hubiera podido visibilizar la violación sexual contra estas personas y, así, alcanzar su condena a futuro.

35. Sentencia del caso Fiscal con Jean Pierre Bemba, párrafo 498. 
Sin perjuicio de lo anterior, en tanto existe la restricción del artículo 7.3, ninguna definición de crimen internacional puede ser realmente neutral, puesto que no alcanza a considerar identidades no binarias.

\section{Referencias}

Berkeley's Gender Equity Resource Center (2016). «Definition of terms». Disponible en http://bit.ly/2cCO6tW.

Butler, Judith (2002). El género en disputa. Buenos Aires: Paidós.

CAMPBELL, Kirsten (2007). «The gender of transitional justice: Law, sexual violence and the International Criminal Tribunal for the former Yugoslavia». The International Journal of Transitional Justice, 1 (3): 411-432. DOI: 10.1093/ijtj/ijmo33.

Centro Nacional de Memoria Histórica (2015). Aniquilar la diferencia: Lesbianas, gays, bisexuales y transgeneristas en el marco del conflicto armado colombiano. Bogotá: CNMH, UARIV, USAID, OIM.

Chang, Iris (1997). The rape of Nanking. Bronx: Basic Books.

Charlesworth, Hilary (1999). «Feminist methods in international law». American Journal of International Law, 93 (2): 379-394. DOI: 10.2307/2997996.

COMISIÓN DE LA VERDAD y RECONCILIACIÓN (2003). Informe final de la Comisión de la Verdad y Reconciliación. Lima.

Comisión Interamericana De Derechos Humanos (2015). «Informe sobre la violencia contra las personas LGBTI en las Américas». OAS/Ser.L/V/II.rev.2. Disponible en http://bit.ly/2vyAKoV.

Comité CEDAW (2010). «Recomendación general 28 relativa al artículo 2 de la Convención sobre la eliminación de todas las formas de discriminación contra la mujer». CEDAW/C/GC/28. Disponible en http://bit.ly/2myt2Kc.

Condorelli, Luigi (1999). «La Cour Pénale Internationale: Un pas de géant (pourvu qu'il soit accompli)». Révue Générale de Droit International Public, 103 (1): 7-21.

Corte Penal Internacional (2016). "Case information sheet. Situation in the Central African Republic. The Prosecutor v. Jean Pierre Bemba». Disponible en http://bit.ly/2f2KFQx.

Cossman, Brenda (2002). "Gender performance, sexual subjects and international law». Canadian Journal of Law and Jurisprudence, 15 (2): 281-296. DOI: 10.1017/ So 841820900003623 .

Defensoría del Pueblo de Colombia (2015). Voces ignoradas: La situación de personas con orientación sexual e identidad de género diversas en el conflicto armado colombiano. Bogotá: Defensoría del Pueblo de Colombia.

FALK, Richard y Quincy WRIGHT (1971). The international law of civil war. Baltimore: John Hopkins.

GARDAM, Judith (2014). «More of the same. Feminist interactions with IHL». Docu- 
mento presentado en Centre for International Governance and Justice Workshop: Recent Developments in Feminist Thinking in International Law and Human Rights. Disponible en http://bit.ly/2uPotvO.

GorRIs, Ellen Anna Philo (2015). «Invisible victims? Where are male victims of conflict-related sexual violence in international law and policy?». European Journal of Women's Studies, 22 (4): 412-427. DOI 10.1177/1350506815605345.

GREY, Rosemary (2014a). "Conflicting interpretations of 'sexual violence' in the International Criminal Court. Recent cases». Australian Feminist Studies, 29 (81): 273-288. DOI: 10.1080/08164649.2014.958123.

-. (2014b). «Sexual violence against child soldiers: The limits and potential of international criminal law». International Feminist Journal of Politics, 16 (4): 601-621. DOI: $10.1080 / 14616742.2014 .955964$.

KAPUR, Amrita y Kelli MudDell (2016). When no one calls it rape. Addressing sexual violence against men and boys in transitional contexts. Nueva York: International Center for Transitional Justice.

Kerry Rittich y otros (1999). «The gender of international law». Proceedings of the Annual Meeting (American Society of International Law), 93: 206-209. Disponible en http://bit.ly/2uZdlh5.

LEATHERMAN, Janie (2012). «Violencia sexual, guerra globalizada y el colapso de los espacios seguros». Politai, 3 (4): 29-43. Disponible en http://bit.ly/2vc8jQY.

Menjívar Ochoa, Mauricio (2001). «Masculinidad y poder». Revista Espiga, 2 (4): 1-8. DOI: 10.22458/re.v2i4.735.

Montoya, Iván (2011). La impunidad en los delitos sexuales cometidos contra niñas, niños y adolescentes del distrito de El Agustino. Lima: Sinco Editores.

Mudrovcic, Zeljka (2001). «Sexual and gender-based violence in post-conflict regions: The Bosnia and Herzegovina case». En The impact of armed conflict on women and girls. A consultative meeting on mainstreaming gender in areas of conflict and reconstruction. Bratislava: UNFPA.

NaCiones Unidas (2001). «Informe del Relator Especial sobre la cuestión de la tortura y otros tratos o penas crueles, inhumanas o degradantes». A/56/156. Disponible en http://bit.ly/2udCwdG.

-. (2016). «Resolución aprobada por el Consejo de Derechos Humanos el 30 de junio de 2016. Protección contra la violencia y la discriminación por motivos de orientación sexual e identidad de género». A/HRC/RES/32/2. Disponible en http://bit. ly/2vZnsTj.

No Tengo Miedo (2016). Nuestra voz persiste: Diagnóstico de la situación de personas lesbianas, gays, bisexuales, transgénero, intersexuales y queer en el Perú. Lima: Tránsito, Vías de Comunicación Escénica.

Oficina del Fiscal de la Corte Penal Internacional (2014). «Policy paper on sexual and gender based crimes». Disponible en http://bit.ly/2vZjvOg. 
Oosterveld, Valerie (1999). «The making of a gender-sensitive International Criminal Court». International Law FORUM du droit international 1 (1): 38-41. DOI: 10.1163/15718049920961729.

-. (2005). «The definition of gender in the Rome Statute of the International Criminal Court: A step forward or back for international criminal justice». Harvard Human Rights Journal, 18: 55-84.

-. (2014). "Constructive ambiguity and the meaning of 'gender' for the International Criminal Court». International Feminist Journal of Politics, 16 (4): 563-58o. DOI: 10.1080/14616742.2014.950818.

SALmón, Elizabeth y Giovanna García (200o). «Los tribunales internacionales que juzgan individuos: El caso de los Tribunales ad hoc para la ex-Yugoslavia y Ruanda y el Tribunal Penal Internacional como manifestaciones institucionales de la subjetividad internacional del ser humano». Derecho y Sociedad, 15: 9-28. Disponible en http://bit.ly/2vhOp7m.

Serrano-Amaya, José Fernando (2014). Chiaroscuro: The uses of «homophobia» and homophobic violence in armed conflicts and political transitions. Tesis doctoral presentada en la Facultad de Educación y Trabajo Social de la Universidad de Sidney. Disponible en http://bit.ly/2udBKh7.

Sivakumaran, Sandesh (2007). «Sexual violence against men in armed conflict». The International Journal of European Law, 18 (2): 253-276. DOI: 10.1093/ejil/chmo13. TAN, Joe (2014). «Sexual violence against children on the battlefield as a crime of using child soldiers. Square pegs in round holes and missed opportunities in Lubanga». Yearbook of International Humanitarian Law, 15: 117-151. DOI: 10.1007/978-90-6704-924-5_6.

Urban WALKer, Margareth (2009). "Gender and violence in focus: A background for gender justice in reparations». En Ruth Rubio-Marin (editora), The gender of reparations. Unsettling sexual hierarchies while redressing human rights violations (pp. 18-62). Cambridge: Cambridge University Press.

\section{Agradecimientos}

Las autoras quisiéramos agradecer a Michelle Reyes Milk y a Karen Anaya por sus inconmensurables aportes y comentarios en la elaboración del presente artículo. Asimismo, la autora Rita Zafra quisiera agradecer a la Beca MISEREOR.

\section{Sobre las autoras}

Rita del Pilar Lucila Zafra Ramos es bachillera en Derecho por la Pontificia Universidad Católica del Perú. Maestría en Derechos Humanos de la Pontificia Universidad Católica del Perú. Investigadora del Grupo de Investigación en Derecho, 
Género y Sexualidad de la Pontificia Universidad Católica del Perú. Su correo electrónico es rita.zafra@pucp.edu.pe.

Pamelhy Isabel Valle Chacaltana es bachillera en Derecho por la Pontificia Universidad Católica del Perú. Su correo electrónico es pamelhy.valle@pucp.edu.pe. 